\title{
REPRESENTACIONES DISCURSIVAS DE ESTUDIANTES DE EDUCACIÓN MEDIA TÉCNICO PROFESIONAL EN EL DISCURSO DOCENTE
}

Discursive representations of secondary vocational schools students in the teachers discourse

MARCELA ROMERO-JELDRES

Universidad Metropolitana de Ciencias de la Educación (Chile) marcela.romero@umce.cl

TRICIA MARDONES NICHI Universidad Metropolitana de Ciencias de la Educación (Chile) tricia.mardones@umce.cl

VALESKA MÜLLER GONZÁLEZ Universidad de Los Lagos (Chile) valeska.muller@ulagos.cl

Resumen

Este estudio da cuenta del análisis crítico del discurso (ACD) realizado en una investigación mayor aplicada a 151 docentes técnicos, que se desempeñan en los niveles diferenciados de liceos de Educación Media Técnico Profesional (EMTP) con alto nivel de vulnerabilidad. Esta fase buscó establecer representaciones discursivas acerca de los estudiantes, cuando los docentes relacionan los conceptos de EMTP, pobreza y familia. El corpus fue analizado desde el modelo Tridimensional de Fairclough. Los resultados reproducen dos discursos sociales, el del Estado y el de la familia, ocultando una dominación al instalar una falsa autonomía social en las poblaciones de estudiantes más pobres, proyectadas en un determinismo, cuando no se ven posibilidades de superar la pobreza.

Palabras clave: Análisis crítico del discurso; recursos lingüísticos; discurso docente; Educación Media Técnico Profesional.

\section{Abstract}

This study gives an account of the critical discourse analysis (ADC) carried out in an investigation, more applied to 151 technical professors, who work in the differentiated levels of secondary vocational schools with high level of vulnerability. This phase sought to establish discursive representations towards students, when teachers relate the concepts of EMTP, poverty and family. The corpus was analyzed from the three-dimensional model Fairclough. The results reproduce two social discourses, that of the state and that of the family, concealing a domination by installing a false social autonomy in the poorest student populations, projected on a determinism, when there is no chance of overcoming poverty.

Key words: Critical discourse analysis; linguistic resources; teacher discourse; vocational/technical secondary education. 


\section{INTRODUCCIÓN}

Por estos días, los liceos técnicos en Chile están siendo requeridos por tres importantes desafíos: fortalecer la formación pedagógica de los profesionales o técnicos de nivel superior, autorizados por la normativa vigente para realizar docencia en la enseñanza media técnico profesional (EMTP ${ }^{1}$ ) (Larrañaga, Cabezas y Dussaillant, 2013); implementar los nuevos cambios curriculares en EMTP, orientados hacia las trayectorias formativas para acceder al trabajo y a la educación superior (Ministerio de Educación de Chile, 2016); y formar como técnicos medios a los estudiantes con el mayor nivel de desventaja social, que sigue la modalidad técnico profesional en la enseñanza media (Sevilla, 2012).

Ortiz (2009) señala que gran parte de los jóvenes de los quintiles más pobres, en vez de desertar, decide continuar estudios técnicos porque sus expectativas de movilidad social o sus necesidades económicas los presionan a buscar trabajo al egresar de la educación media.

Por consiguiente, esta investigación ha buscado develar las representaciones discursivas planteadas por los profesores de EMTP, en tanto creencias o imágenes construidas mediante el lenguaje y materializadas por medio del léxico y los elementos semánticos, evidenciando las relaciones de saber y poder que se dan entre los profesores de EMTP y sus estudiantes, y la acción pedagógica que reproduce, estratifica y legitima a los individuos persuadiéndoles de que ello no es social, sino natural (Vasilachis de Gialdino, 2003).

\section{FUNDAMENTACIÓN TEÓRICA}

El Análisis Crítico del Discurso (ACD) es un enfoque con una perspectiva crítica ante la realización del saber. Se centra en lo social, especialmente en el rol del discurso de la producción del poder o dominación, usado para legitimar grupos hegemónicos al controlar el texto y el contexto (Van Dijk, 2016). El ACD entiende el discurso como acontecimiento o acto comunicativo que, implícita o de manera manifiesta, porta ideologías, creencias, actitudes, entre otros elementos. Para los autores que trabajan con el ACD, las ideologías forman la base de los sistemas de creencias o representaciones sociales de grupos específicos y, como tal, corresponde a una estructura esquemática de los grupos sociales que opera manteniendo las relaciones desiguales del poder (Van Dijk, 2001; Wodak, 2016).

En esta perspectiva, el discurso sería ideológico en la medida que favorece la conservación de las relaciones de poder y dominación (Fairclough, 2001), al mismo

\footnotetext{
${ }^{1}$ La sigla EMTP permite identificar a los liceos de enseñanza media técnico-profesional, y a los liceos polivalentes y politécnicos que imparten tanto la modalidad científico-humanista como la técnica-profesional. 78 | Alpha No 52 (JuLIO 2021) PÁGS. 77-89. ISSN 07 16-4254
} 
tiempo que comparten la idea de que la ideología se materializa por medio del lenguaje, ya que "todo lo ideológico posee significado: representa, figura o simboliza algo que está fuera de él" (Voloshinov, 1976, p.14-21), es decir, sería un signo ideológico y, como tal, un segmento material de realidades, posibles de corporeizar materialmente y de estudiar.

Ahora bien, desde el ámbito de la interacción y contexto social, es posible observar que el espacio educativo se devela como un instrumento de reproducción del orden social, con un discurso pedagógico que se caracteriza por ser reglamentado, tanto para el profesorado como para los estudiantes. Según la OCDE (2004), "la educación chilena está influenciada por una ideología que da una importancia indebida a los mecanismos de mercado para mejorar la enseñanza y el aprendizaje" (p. 290). Frente a este sistema educativo, Cornejo (2006) señala que ello ha generado, a la fecha, "escuelas empobrecidas a las cuales asisten niños y jóvenes de sectores marginados y con escaso capital cultural, en las cuales resulta mucho más difícil enseñar y aprender y que cuentan (como contradicción máxima,) con docentes en peores condiciones de trabajo" (p.125).

Por su parte, Sleeter, Montecinos y Jiménez (2016) enfatizan que esta segregación de escuelas por clase social ha ido en aumento en los últimos 40 años. Se observa en el ejercicio docente actitudes negativas y desesperanzadas respecto del éxito académico de estudiantes provenientes de contextos sociales de pobreza. Esto resulta importante para este estudio, al visualizar si este discurso también se reproduce en docentes técnicos con desempeño en los niveles diferenciados de las especialidades TP cuya situación laboral no ha sido tan visible desde el campo de la pedagogía, por desempeñarse en una modalidad de educación secundaria, en el sentido de su oficio y de su práctica y bajo una normativa del Ministerio de Educación de Chile (2011) que les permite impartir asignaturas en los niveles diferenciados de las especialidades TP, sin ser docentes titulados en pedagogía. Por tanto, el profesor que se desempeña en el área TP construye su saber pedagógico sobre su propio desempeño, desde una condición subalterna, donde la enseñanza tiene dos caras: la de producción, en tanto designa momentos de relaciones, cambios y posibilidades y, una segunda cara de exclusión por cuanto muestra la descalificación del saber pedagógico y sus repercusiones en el profesorado (Zuluaga, 2005).

\section{MARCO METODOLÓGICO}

El estudio forma parte de una investigación mayor, que se emprende desde una investigación mixta. Este artículo da cuenta de la incrustación de una pregunta abierta en un instrumento acerca del dominio de competencias pedagógicas en Educación Media Técnico Profesional. El anidado posibilitó la conformación de un corpus que da cuenta de las relaciones discursivas posibles de profesores técnicos y su vínculo con los estudiantes, cuando sus voces se sitúan sobre tres conceptualizaciones problemáticas, presentes en el cotidiano laboral como son la EMTP, la pobreza y la familia. 
Los participantes fueron 151 profesores técnicos, sin formación pedagógica que se desempeñaban en los niveles diferenciados de 54 liceos de Educación Media Técnico Profesional (EMTP) con alto nivel de vulnerabilidad, pertenecientes a 9 comunas de la zona sur de Santiago (Buin, Calera de Tango, El Bosque, La Cisterna, Lo Espejo, La Granja, Paine, San Bernardo, San Ramón). La muestra de investigación fue tomada en forma aleatoria y calculada según los planteamientos de Hulland, Chow y Lam (1996) que sugieren una muestra mínima de 100 encuestados.

\subsection{PROCEDIMIENTO}

La investigación se fundó en el análisis crítico del discurso (ACD) (Fairclough, 1989; Wodak y Fairclough, 1997; Van Dijk, 2001) examinando las huellas lingüísticas y perspectivas semánticas del discurso docente. Respecto de la huella de la enunciación se consideró el análisis a partir de la denominada teoría de la enunciación desarrollada por Benveniste (2017). Por tanto, el análisis del corpus se trató separando la oración del enunciado y la enunciación del enunciado, identificando el papel que desempeñan los docentes en el proceso de enunciación.

Siguiendo las dimensiones discursivas establecidas en los tres estadios del ACD planteados por Fairclough (1989), la propuesta analítica optó, en una primera instancia, por enfocarse en el análisis descriptivo de las representaciones discursivas de los estudiantes de EMTP presentes en el discurso, considerando el ámbito del significado global, es decir, en las macroestructuras semánticas (Van Dijk, 2001), y el ámbito del significado local. Para tal efecto, se diseñaron matrices en las que se introdujeron unidades lingüísticas de análisis. Para el significado global, se consideraron unidades léxicas del discurso y para el significado local componentes de base gramatical. En segunda instancia, se produce la interpretación de las relaciones entre los procesos del discurso, estableciendo inferencias semánticas. Por último, se establece el análisis crítico que se caracteriza por trascender el discurso, considerando el contexto de la producción discursiva, dando explicación a la correspondencia entre procesos discursivos y procesos sociales. En esta etapa, el o la analista recurre al empleo de las teorías sociales como señalan Wodak y Fairclough (1997).

\section{RESULTADOS Y DISCUSIÓN TEÓRICA}

\subsection{MACROESTRUCTURA SEMÁNTICA DEL DISCURSO}

El análisis arrojó tres temas relevantes que fueron identificados en el discurso de los docentes técnicos. El primer tema asociado a la representación discursiva de los estudiantes en el discurso de los docentes técnicos es la pobreza. En el discurso docente, se aprecian descripciones acerca de los estudiantes de EMTP, relacionándolos con condiciones de carencias socioeconómicas. En el ámbito enunciativo, los recursos léxicos evidencian las 80 | Alpha No 52 (Julio 2021) PÁGs. 77-89. ISSN 07 16-4254 
relaciones entre la carencia de recursos económicos y la pobreza, porque estos estudiantes "no tienen recursos" (E5) y "coincide normalmente que alumnos de EMTP viven en una realidad de pobreza" (E 233).

Sin embargo, el término pobreza también se relaciona desde una perspectiva semántica con el término vulnerable, segundo tema evidenciado en el discurso docente. Se describe a los estudiantes EMTP como pobres, producto de las carencias económicas, y también como vulnerables, por carecer de comportamientos sociales, pautas de crianza o de maneras comportamentales de las emociones siendo muchas veces etiquetados de forma negativa por ello. Dichas carencias se observan en enunciados que establecen que estos son estudiantes "provenientes de hogares destruidos por un círculo cerrado de pobreza, familias disfuncionales con hijos abandonados, carentes de afecto que lo buscan en liceos públicos y que son aquellos donde descargan su ira, rebeldía, resentimiento, frustración" (E3).

El tercer tema corresponde a la posibilidad que tienen los estudiantes de EMTP de ingresar al mundo laboral gracias al estudio en establecimientos Técnicos Profesionales. Según el discurso docente, este tipo de establecimientos educacionales entrega a sus estudiantes herramientas para adentrarse al mundo laboral, considerando que provienen de ámbitos sociales vulnerables y de pobreza. Por tal motivo, la EMTP sería una oportunidad para salir de la situación socioeconómica deprimida en la que se encontrarían, al tener la posibilidad de ingresar al mundo laboral, como plantea E 46 al señalar que "fundamentalmente creo que es una gran oportunidad que los jóvenes tengan acceso a una educación técnico profesional por las oportunidades laborales que les puede entregar este tipo de educación".

Esta imagen acerca de los estudiantes de EMTP, caracterizados en un ámbito de pobreza y de vulnerabilidad social que pueden adentrarse al mundo laboral mediante la Educación Técnico Profesional (ETP), presenta concordancias con los discursos de los organismos nacionales asociados a ella, al mismo tiempo que reproduce un discurso filantrópico, de un Estado que organiza la pobreza al invertir en gasto público. Desde esta perspectiva, el discurso docente reproduciría significados establecidos en el ámbito de las políticas públicas nacionales. Volveremos a esta idea más adelante.

\subsection{SIGNIFICADO LOCAL DEL DISCURSO}

\subsubsection{ACERCA DE LA INTENSIFICACIÓN}

Respecto de la intensificación, se observó en las representaciones discursivas en los estudiantes pertenecientes a EMTP una predominancia de enunciados que producen un realce lingüístico (Vigara, 1992). La vulnerabilidad remite a la experiencia de estar expuesto a; no obstante, también se la asocia con la pobreza en la expresión vulnerable, estableciendo un discurso que la enmarca con la situación de extrema pobreza en la que 
se encontrarían los escolares que asisten a estos establecimientos educativos. Ahora bien, el intensificador predominante es el adverbio más que se presenta antecediendo al adjetivo vulnerable, acentuando la característica de los estudiantes de estos establecimientos, como se observa en el enunciado de E283: "En cuanto a la pobreza siempre está asociada a la EMTP, ya que van los alumnos más vulnerables y también su familia" o en el de E624: "un gran porcentaje de los alumnos de nuestro país estudian en este sistema y son los más vulnerables". Asimismo se puede apreciar que el adverbio más también es utilizado para incrementar la valoración de la formación técnica, como por ejemplo en E367: "permiten a los alumnos en un corto periodo acercarse al mundo laboral en buenas condiciones lo que los hace mucho más valorados".

Desde el análisis, se distingue el uso de la intensificación en el discurso docente dirigido a reforzar los tres grandes temas ya señalados. Los estudiantes de EMTP no solo son pobres y vulnerables, sino los más pobres y vulnerables; y la educación TP tiene el poder de convertirlos en más valorados. En este sentido, la formación técnica es evaluada como un potente agente transformador.

\subsubsection{ENCUANTO A LA ATENUACIÓN}

Respecto de otras huellas lingüísticas, el corpus devela la atenuación como categoría pragmática, cuyo valor en lo textual busca minimizar, suavizar o reducir la fuerza de las representaciones discursivas que los docentes construyen en los estudiantes de EMTP. En un primer análisis, se puede observar atenuadores como: la mayoríalgran parte/muchos/muchas veces, que hacen referencia a los estudiantes, pero sin incluirlos a todos, es decir, suavizando, por ejemplo, la cantidad de escolares que no logran mejores aprendizajes o la cantidad de escolares pobres que estudian en la EMTP, como se aprecia en las palabras de E7: "la mayoría no logra mejores aprendizajes porque existe una conciencia de que ellos están determinados a seguir en su situación [...], esta mentalidad reina en la mayoría de los estudiantes y apoderados". La afirmación aparece atenuada porque involucra un determinismo del que el hablante no tiene certeza. De cualquier forma, en este caso la responsabilidad respecto del mejoramiento en su preparación recae en los propios estudiantes y apoderados.

En un segundo análisis, se puede encontrar que los atenuadores muchos/la gran mayoría son utilizados para valorar la decisión de los estudiantes por la EMTP frente a sus condiciones de vida. En este caso, el atenuador opera minimizando los resultados, de modo que la evaluación positiva se entiende no para todos los estudiantes, como señala E252: "muchos optan por esta, para ayudar a sus familias y entrar al mundo del trabajo"; y señala E246: "la gran mayoría de nuestros estudiantes logran integrarse al mundo laboral exitosamente".

El recurso de la atenuación permite mitigar posibles acusaciones al sistema educativo, por no cumplir siempre con las expectativas de los estudiantes. En este punto 82 | Alpha No 52 (Julio 2021) PÁGs. 77-89. ISSN 07 16-4254 
aparece una alternativa frente a la posibilidad de insertarse en el mundo laboral. Esto es, los estudiantes podrían continuar estudios superiores, pero, de nuevo, esta vía no es posible para todos, como se observa en E352: "Hay muchos alumnos que debido a la pobreza no pueden proyectarse a estudiar en la universidad"y en E353: "ya que sus horizontes de posibilidades para entrar a una universidad, muchas veces, sobrepasa la capacidad adquisitiva de familias que se encuentran en condición de pobreza". Estos ejemplos develan la presencia constante de atenuadores con el fin de no generalizar al referirse a la condición de vida de los estudiantes de EMTP, a sus expectativas y el logro de estas.

\subsubsection{DELOS EPÍTETOS}

Siguiendo a Demonte (1982), se buscó en el corpus juicios de valor que calificaran a los estudiantes por medio de opiniones explicativas, descriptivas, restrictivas. En relación con lo descriptivo y las condiciones de procedencia de los estudiantes, el discurso docente da cuenta de tres focos de opinión. Un primer foco asociado con la descripción de las características sociales de los estudiantes como, por ejemplo, E 522: "alumnos, con una cantidad enorme de vicisitudes, con problemas conductuales y psicológicos, cognitivos". Un segundo foco de opiniones asociadas con explicaciones acerca de la superación de la pobreza, como por ejemplo lo enunciado por E 354: "Estos logros alcanzados por los alumnos, sin duda será una oportunidad para mejorar la calidad de vida de muchas familias". Un tercer foco asociado con lo restrictivo, cuando se plantean posibilidades de continuidad de estudios, por ejemplo, lo señalado por E 262: "el nivel de ingresos que obtiene un estudiante recién egresado de esta modalidad no ayudará a superar dicha condición, es más, muchas veces la reproduce debido al poco espacio para el desarrollo de la visión futura de los estudiantes, necesaria para la continuidad de estudios".

El análisis evidencia que los epítetos utilizados muestran la visión de los docentes acerca de la realidad problemática de los estudiantes y las dificultades ante la prosecución de estudios. Sin embargo, ante este panorama, la EMTP es sentida como una vía de mejoramiento.

\subsubsection{DEL POSICIONAMIENTO ENUNCIATIVO}

El posicionamiento de los docentes de EMTP en su discurso se configura desde un nosotros que es planteado para hacer ver lo que hacen por los estudiantes. El ellos, en tanto, se configura como un deíctico negativo, que da cuenta de que los estudiantes no funcionan ni como locutores ni como alocutarios, lo que puede ser observado en el enunciado de E 522: "Nosotros les damos las herramientas para que ellos puedan desarrollarse, en forma activa en el mundo laboral"; o en el enunciado de E 522: "Y nos sentimos orgullosos cuando ellos egresan de esta modalidad de enseñanza y obtienen su título profesional". 
Respecto de este posicionamiento, es posible plantear tres cuestiones: la primera, asociada a una toma de distancia por medio del recurso a la impersonalidad, para relevar un problema en el que los estudiantes son vistos por sus propias familias como pobres, no solo en recursos económicos, sino también en inteligencia como se aprecia en E502: "Se piensa que los jóvenes pobres estudian en liceos Técnico Profesionales. Y las familias $\underline{\text { creen que los hijos que no son inteligentes deben estudiar en un liceo TP y que en sus }}$ expectativas no está el ingreso a la universidad por falta de recursos económicos".

La segunda, asociada a una toma de posición declarativa que devela el pensamiento del profesor. En este sentido, lo que declara correspondería a la realidad de la que puede dar cuenta. Esta vez la explicación está en la situación socioeconómica de la familia como señala E351: "Pienso que nuestros alumnos de enseñanza media de colegios municipales generalmente estudian en colegios EMTP, porque sus familias no cuentan con mayores recursos".

La tercera cuestión está asociada a una toma de posición declarativa que da cuenta de las creencias del pensamiento del profesor. En este sentido, lo que declara es un supuesto que podría no corresponder a la realidad, es decir, creo involucra una aserción mitigada que convierte en una enunciación subjetivada el hecho afirmado (Benveniste, 2017), como se observa en E37: "Creo que la EMTP es una solución a una búsqueda de las familias más vulnerables al problema de la pobreza".

Este análisis confirma lo expuesto hasta aquí, en relación con las características de los estudiantes y sus familias y el grado de incerteza que manifiestan los docentes respecto de los motivos de elección de la EMTP.

\subsection{EL DISCURSO DOCENTE DESDE LA PERSPECTIVA INTERTEXTUAL Y SOCIAL}

El análisis de los elementos de significado global y local del discurso docente posibilita avanzar hacia el análisis intertextual. La interpretación de las relaciones entre el corpus estudiado y la interacción social permite plantear que el discurso docente entiende la pobreza desde un enfoque multidimensional. Es decir, reproduce los planteamientos del PNUD (1997) que se refieren a la pobreza como aquella situación en la que "se deniegan las oportunidades y las opciones más fundamentales del desarrollo humano: vivir una vida larga, sana, creativa y disfrutar de un nivel decente de vida, libertad, dignidad, respeto por sí mismo y por los demás" (p. 17). Los índices de pobreza asociados a los liceos técnicos, teniendo como base la caracterización de los estudiantes prioritarios que ingresan a la EMTP, sirven de fundamento a esta representación. Siguiendo a Martucelli (2017), el discurso docente clasifica la vulnerabilidad de los estudiantes desde una semántica voluntarista, al relacionarla con la exclusión o pobreza y, por tanto, semánticamente sería inaceptable a ojos del progreso, lo que hace preciso regularla e incluso extirparla. 
Del corpus del discurso docente se infiere un vínculo existencial entre el sistema de EMTP y las expectativas de las familias debido a la proveniencia de los estudiantes y el escenario educativo. A este respecto, estudios nacionales señalan que el rendimiento académico previo no incide en la elección de un establecimiento al interior de la EMTP, ni la condición económica de los estudiantes en octavo año básico (según puntajes en el SIMCE), por cuanto es bastante homogénea entre tipos de establecimientos. Por el contrario, son las familias las que en su mayoría esperan que sus hijos e hijas continúen estudios universitarios y por esa razón los matriculan en la enseñanza humanista científica, mientras que quienes esperan que los hijos sigan estudios en Centros de Formación Técnica o Institutos Profesionales o que no prosigan estudios superiores, se inclinan en forma mayoritaria por la modalidad técnico profesional (Larrañaga et al., 2013) y, en tal caso, los docentes de EMTP reproducen el discurso de las familias.

En relación con la valoración de la educación técnica, el discurso docente reproduce lo planteado por los informes de organismos internacionales (OCDE, 2010) que señalan que la Educación Técnica Profesional (ETP) ofrece la oportunidad de acceder a un sistema educativo fuertemente orientado a una actividad laboral e industrial que aporta a la disminución del desempleo juvenil. No obstante ello, el discurso docente, se presenta atenuado y con ciertos criterios de realidad, que los distancia. Ello se explica desde investigaciones que se han llevado a cabo en Chile, que señalan que los egresados de la EMTP presentan leves diferencias a favor de la EMTP, donde las ventajas influirían en la identificación más temprana con el mundo del trabajo y su voluntad de participar en él, en comparación con sus pares del área humanística-científica que presentan mayor tendencia a la inactividad (Ortiz, 2011, p. 194).

Una segunda atenuación respecto del empleo, podría explicarse desde el sistema de educación dual (educación en la empresa) que opera con bastante éxito en Alemania, siendo el único país que no presenta un desempleo relativo mayor entre los jóvenes, que optan por laETP, respecto de la educación "tradicional", ello porque el sector público alemán también actúa como un empleador de último recurso de los jóvenes evitando el desempleo de aquellos que son menos educados (Beyer, 1998, p. 92-93). Esta realidad es por completo distinta en Chile. Ortiz (2009) señala que la formación TP sigue siendo un ciclo terminal de estudios para los más pobres, que forjaría ilusiones ocupacionales; no obstante su alta demanda introduce un dilema asociado más con una política de equidad (p. 114).

Sumado a lo anterior, los discursos docentes son coherentes con los estudios nacionales que plantean que el nivel de rezago que tienen los estudiantes de EMTP para seguir estudios respondería a que la mayoría de los jóvenes empieza a trabajar antes de ingresar a la educación superior por razones de la precaria condición económica de sus hogares (Larrañaga et al., 2013). Existe en el discurso de los docentes una constatación de las características de la pobreza, conceptualizadas desde las definiciones que plantea el PNUD (1997) y Sen (2000), asimismo, proponen un discurso asociado a la superación 
de la pobreza por medio de la EMTP similar al que presenta la OCDE (2010), no obstante, el texto presentado es de corte restrictivo hacia los estudiantes, ya que no todos podrían alcanzar estudios superiores.

Así, el análisis crítico del corpus coloca en evidencia la manera en que los discursos docentes reproduciría la ideología del progreso, asociándola de manera directa con la modernidad, reflejada desde los alocuciones de los organismos internacionales como la OCDE y el PNUD, institucionalizados por el Estado chileno; al mismo tiempo, en un plano más cercano al aula, el discurso se torna garantista respecto de la idea de progreso establecido en las expectativas familiares y la empleabilidad, donde la EMTP surge como la gran articuladora del destino de los estudiantes pobres para la mejora de su porvenir, asumiendo discursos cuyos resultados foráneos no siempre son coincidentes con las condiciones materiales, sociales y económicas que se dan en otras latitudes y sus beneficios. Esta ideología de progreso sigue ocultando una dominación al instalar una falsa autonomía social en las poblaciones más pobres, cuyo deseo de un renovado tiempo histórico se mantiene en una situación de postergamiento permanente. En este sentido el discurso docente reproduce dos discursos sociales, el del Estado y el de la familia.

\section{CONCLUSIONES}

Las representaciones discursivas acerca de los estudiantes de EMTP, planteadas por los profesores de EMTP y materializadas por medio del léxico y los elementos semánticos, develan dos líneas discursivas. La primera está asociada a una imagen que los retrata como estudiantes "víctimas" de nacer en condiciones de vida pobres y vulnerables, cuyas familias deciden su ingreso a la EMTP como un paliativo para extirpar la vulnerabilidad o mejorar las condiciones de vida.

Esta primera línea muestra cómo se reproduce el discurso de pobreza multidimensional planteado por el PNUD (1997) y Sen (2000), asociado con la privación de capacidades y efectos relacionados con el daño psicológico, la pérdida de motivación para trabajar, de cualificaciones y de confianza en uno mismo, aumento de enfermedades, perturbación de las relaciones familiares y de la vida social, aumento de la exclusión social, empeoramiento de las tensiones sociales y de la asimetría entre los sexos. En este corpus, el discurso se reproduce por medio de etiquetas de connotaciones negativas (ira, rebeldía, resentimiento, frustración, retraso) más que como denuncia para la restitución de la justicia social, hacia la existencia de los sentidos de los estudiantes.

En la misma idea, también es posible inferir un vínculo existencial entre la EMTP y las expectativas de las familias. El discurso docente reproduce la deseabilidad familiar, en tanto, "producir un sujeto", salvado de la vulnerabilidad por medio de la técnica. En este discurso la perspectiva de la vulnerabilidad, planteada por Martucelli (2017), explica una semántica voluntarista que la pone en cuestión, como inaceptable frente al progreso $\mathrm{y}$, por tanto, asume la deseabilidad de una acción colectiva entre familia y EMTP, en pos 86 | ALPHA No 52 (Julio 2021) PÁGs. 77-89. ISSN 07 16-4254 
del bienestar, cuestión que resulta también una oportunidad para que el discurso docente se instale en un rol de vigilante de lo familiar, por cuanto sin su apoyo, extirpar la vulnerabilidad resulta imposible.

La segunda línea discursiva se asocia con el potencial que se le asigna a la Educación Técnica como un medio para romper el círculo con la pobreza, mejorar los ingresos de los estudiantes y sus familias y proseguir estudios universitarios. Esta línea reproduce el enfoque de la pobreza basada en la renta, debido a que sus efectos se asocian con el reparto entre todos y cada uno de los factores productivos, y donde cada familia vería incrementado los recursos económicos del hogar y, por tanto, el discurso docente plantea tres énfasis: uno valorativo para la EMTP, reproductor de lo señalado por países que han superado el desempleo juvenil por medio de la Educación Técnica (Bayer, 1998); un segundo discurso atenuado, pero disidente, respecto de informes internacionales y las bondades ofertadas, para "extirpar la vulnerabilidad" y, por último, un tercer discurso restrictivo hacia los estudiantes que da cuenta del nivel de rezago que tienen los estudiantes para seguir estudios, ya que empiezan a trabajar antes de ingresar a la educación superior por razones de la precaria condición económica de sus hogares (Larrañaga et al., 2013). En ese sentido, la imagen proyectada final está asociada con el determinismo, cuando no se ven posibilidades para la superación de la pobreza.

En todo caso, desde la perspectiva del ACD, se puede señalar que las relaciones de poder establecidas en este discurso docente, reproduce discursos hegemónicos respecto de los estudiantes pertenecientes a la EMTP. Las prácticas lingüísticas de los docentes portarían creencias y estereotiparían a estos estudiantes en las representaciones discursivas, plasmando en ellas las desigualdades sociales mediante el uso del lenguaje y, por esta razón, mantendrían las relaciones desiguales de poder. Desigualdad que estaría contemplada en las representaciones discursivas a los estudiantes de la EMTP cuando los caracterizan como pobres y vulnerables (desde la estructura familiar), con capacidades cognitivas básicas o descendidas, como consecuencia del medio o el origen social y, en casos contrarios, con dificultades de acceso a la educación superior por falta de recursos. En consecuencia, el discurso docente invisibilizaría a las y los estudiantes de EMTP en su potencial de aprendizaje, construyendo lingüísticamente la huella de la desigualdad social en relaciones de poder y dominación desiguales.

Por lo anterior, es importante preguntarse por la situación presente de los docentes técnicos. Es posible que, al mantener un rol profesional subalterno, por no tener estudios de pedagogía, y al estar insertos en comunidades escolares con docentes pedagogos profesionales, reproduzcan huellas culturales del país que subvaloran la educación técnica, al mismo tiempo que ignoran a los estudiantes pobres, respectos de sus posibilidades de desarrollo social y laboral. En todos los casos resultaría importante saber si en efecto se oponen activamente a sus discursos o los resisten pasivamente. 
Se agradece el financiamiento de esta investigación a CONICYT por medio del Proyecto Fondecyt de Iniciación No. 11140650.

\section{OBRAS CITADAS}

Benveniste, Émile (2017). Artículos fundadores. De la subjetividad en el lenguaje. En M. C. Pereira, Semiología.Cuadernillo 2. En torno al análisis de los discursos. Buenos Aires: Universidad de Buenos Aires, 6-12.

Beyer, Harald (1998). ¿Desempleo juvenil o un problema de deserción escolar? Centro de Estudios Públicos Nº 277.

Cornejo, Rodrigo (2006). El experimento educativo chileno 20 años después: Una mirada Crítica a los logros y falencias del sistema escolar. Revista Electrónica Iberoamericana sobre Calidad, Eficacia y Cambio en Educación. REICE 4(1), 118-129.

Demonte, Violeta (1982). "El falso problema de la posición del adjetivo: dos análisis semánticos". Boletín de la Real Academica Española, 62(227), 453-486.

Fairclough, Norman (2001). "The dialectic of discourse". Textus, 14(2), 231-242.

(1989). "Discourse as social practice". En N. Fairclough, Language and Power. Londres y Nueva York: Longman, 17-42.

Hulland, John; Chow, Yiu Ho y Lam, Shunyin. (1996). "Use of causal models in marketing". International. Journal of Research in Marketing, 13(2), 181-197.

Larrañaga, Osvaldo; Cabezas, Gustavo y Dussaillant, Francisca (2013). Informe completo del Estudio de la Educación Técnico Profesional. Santiago: Programa de las Naciones Unidas para el Desarrollo. Chile. Área de Reducciòn de la Pobreza y la Desigualdad.

Martucelli, Danilo (2017). "Semánticas históricas de la vulnerabilidad". Revista de Estudios Sociales (59), 125-133.

Ministerio de Educación de Chile (2016). Ley 20903.Politica Nacional Docente. Santiago: Biblioteca del Congreso Nacional de Chile. Disponible en http://bcn.cl/22srj

— (2011). Educación Técnica Profesional en Chile; Antecedentes y Claves de Diagnóstico. Santiago: Centro de Estudios. División de Planificación y Presupuesto.Ministerio de Educación.

OCDE. (2010). Synthesis Report of the OECD Reviews of Vocational.Learning for Jobs. París: Secretary General of the OECD.

— (2004). Revisión de políticas nacionales de educación. París: OECD. 
Ortiz, Ivan (2011). "Situación ocupacional de los jóvenes egresados de la educación media: comparación entre los egresados de la formación técnico-profesional y la humanista-científica". Estudios Pedagógicos Vol. 37 № 2, 181-196.

_ (2009). “Es relevante la educación media técnico profesional?” Persona y Sociedad, 23(3), 99-115.

PNUD (1997). Human Development Report 1997. New York: Oxford University Press,

Sen, Amartya (2000). Desarrollo y Libertad.Capitulo 4, La pobreza como privación de capacidades. Buenos Aires: Editorial Planeta S.A.

Sevilla, María Paola (2012). Educación técnica profesional en Chile. Antecedentes y Claves de Diagnóstico. Santiago: Centro de Estudios MINEDUC.

Sleeter, Christine; Montecinos, Carmen \& Jiménez, Felipe (2016). "Prepararing Teachers for Social Justice in the Context of Education Policies that Deepen Class Segregation in Schools: The Case of Chile". En J. Lampert, \& B. Burnett (Edits.), Teacher Education for High Poverty Schools (págs. 171-191). New York: Springer Book Series.

Van Dijk, Teun A. (2016). "Análisis Crítico del Discurso". Revista Austral de Ciencias Sociales, 30, 203-222.

— (2001). "Multidisciplinary CDA: a plea for diversity". En R. Wodak, \& M. Meyer, Methods of critical discourse analysis. (1 ed.). London: Sage, 95-120.

Vasilachis de Gialdino, Irene (2003). Pobres, pobreza,identidad y representaciones sociales. Barcelona: Gedisa.

Vigara Tauste, Ana María (1992). Morfosintaxis del español coloquial. Esbozo estilístico. Madrid: Gredos.

Voloshinov, Valentín (1976). El estudio de las ideologías y la filosofía del lenguaje. El signo ideológico y la filosofía del lenguaje. Buenos Aires: Nueva Visión.

Wodak, Ruth (2016). The discourse historical approach. En R. Wodak, \& M. Meyer, Methods of critical discourse analysis. (3 ed.). London: Sage, 24-58.

Wodak, Ruth \& Fairclough, Norman. (1997). "Critical Discourse Analysis". En T. A. van Dijik, Discourse as Social Interaction. London: Sage, 258-284.

Zuluaga Garcés, Olga Lucía (2005). "Foucault: Una lectura desde la práctica educativa. En U. P. Nacional-IDEP”, Foucault, la Pedagogía y la Educación. Pensar de otro modo. Bogotá: Cooperativa Editorial Magisterio, 11-37. 